\title{
Corrosión de armaduras embebidas
en hormigón carbonatado(")
}

C. ANDRADE $(* *)$, S. AI.GABA y J. A. GONZAILEZ (***)

\section{INTRODUCCION}

Los componentes ácidos de la atmósfera, en particular el $\mathrm{CO}_{2}$, pueden neutralizar (carbonatar) el electrólito que llena los poros del hormigón y las fases del clínker, transformándolos en variedades del $\mathrm{CO}_{3} \mathrm{Ca}$.

La carbonatación no sólo modifica la estructura, de la pasta de cemento y por lo tanto sus propiedades, sino que también tiene una influencia decisiva sobre la velocidad de corrosión de las armaduras (1) (2). En un reciente informe del Comité 12-CRC de la RILEM (3) se afirmaba que: "si se exceptúa la corrosión causada por $\mathrm{Cl}^{-}$, actualmente se está de acuerdo en que la carbonatación del hormigón es la condición esencial para la corrosión de las armaduras". (Aquí se debería añadir que esto se aplica a los hormigones simplemente armados, pues en los pretensados también es muy peligroso el fenómeno conocido como corrosión bajo tensión).

La carbonatación puede desencadenar el proceso de corrosión, debido a que el descenso de $\mathrm{pH}$ que provoca hace posible el paso de las armaduras del estado pasivo al activo. La velocidad de ataque depende, sin embargo, de otros factores externos: un contenido mínimo de humedad en el medio ambiente que garantice la existencia del electrólito en los poros del hormigón, y un libre acceso de $\mathrm{O}_{2}$ hasta la superficie metálica para que pueda producirse la semirreacción catódica.

Numerosos autores están de acuerdo con estos puntos, pero sin aportar pruebas cuantitativas de los mismos. Es objeto principal de este trabajo contribuir a llenar este vacío.

La escasez de datos cuantitativos se explica por las especiales características del medio, que incrementan en gran medida la laboriosidad de los métodos gravimétricos tradicionales de estudio de la corrosión y plantean, por otra parte, serias dificultades (elevada resistividad e imposibilidad de observación directa de los electrodos) al empleo de las técnicas electroquímicas (4) introducidas en este campo en 1959 por Kaesche (5), Baümel (6) y Engell (7). Estas dificultades han hecho que, estos métodos electroquímicos, no se hayan usado en el hormigón tan exacta y ampliamente como en otros materiales. Y cuando han sido aplicados, o se han ignorado las limitaciones, obteniendo resultados erróneos, o se

\footnotetext{
(*) Este artículo ha sido publicado en la Revista "British Corrosion Journal" vol 15 n." 3 (1980), página 135, y se reproduce aquí por la gentileza de sus editores: The Metals Society (Londres).

(**) C. Andrade - Dr. en Química Industrial - Instituto Eduardo Torroja - C.S.I.C. - Madrid.

(***) J. A. González y J. Algaba - Dr. en Quimica Industrial y Lcdo. en Químicas respectivamente - CENIM - C.S.I.C. - Madrid. 
ha usado exclusivamente disolución saturada de $\mathrm{Ca}(\mathrm{OH})_{2}$ o extractos de cemento, que aunque sus propiedades termodinámicas son similares a las del hormigón, las características cinéticas que promueven en el acero son muy diferentes.

Además, tampoco se contaba con una técnica de medida de las variables cinéticas del proceso de corrosión de las armaduras rápida y fiable, por lo que los resultados se expresan de forma imprecisa con observaciones visuales o bien con la simple indicación del potencial de corrosión, $E_{\text {corr }}$, cuyas limitaciones para aportar indicaciones cinéticas cuantitativas son bien conocidas (4).

En esta investigación se han utilizado medidas de la Resistencia de Polarización, $R_{\mathrm{p}}$, técnica desarrollada por Stern y colaboradores (8) (9), para evaluar la intensidad de corrosión, $I_{\text {corr }}$, de las armaduras.

\section{METODO EXPERIMENTAL}

\section{Materiales}

Los redondos usados para los ensayos fueron de acero de alta resistencia, estirado en frío, de $7 \mathrm{~mm}$ de diámetro y $8 \mathrm{~cm}$ de longitud. Las barras fueron pulidas con papel abrasivo hasta el n. ${ }^{\circ} 00$, lavadas con agua y desengrasadas en acetona. La interfase mortero-aireacero fue aislada con una cinta adhesiva. La superficie de ataque fue de $10 \mathrm{~cm}^{2}$.

El mortero fue preparado de acuerdo con la norma española RC-75 (10) con una relación $a / c$ de 0,5 y una relación cemento/arena de 1/3. El cemento usado fue un P-350.

Las probetas de $2 \times 5,5 \times 8 \mathrm{~cm}$ tenían embebidas dos barras idénticas. Fueron curadas en una atmósfera del $90 \%$ de HR y a $20 \pm 2^{\circ} \mathrm{C}$ durante las primeras $24 \mathrm{~h}$, y luego hasta 28 días en la atmósfera del laboratorio con una HR de alrededor del $50 \%$.

El mortero fue fabricado sin aditivos, con un $2 \%$ de $\mathrm{Cl}_{2} \mathrm{Ca}$ como agente corrosivo y $3 \%$ de $\mathrm{NO}_{2} \mathrm{Na}$ como inhibidor de corrosión (todas las proporciones son en relación al peso de cemento).

\section{Experimentos}

Con cada tipo de aditivo se fabricaron cuatro probetas idénticas. Después de los 28 días de curado, dos probetas fueron carbonatadas en una cámara saturada de $\mathrm{CO}_{2}$ con $\mathrm{HR}-50 \%$ (estas condiciones son las que más aceleran la carbonatación del hormigón) (1). Las otras dos probetas se guardaron como control.

Cuando la carbonatación fue completa, todas las probetas (control y carbonatadas) fueron sometidas en sucesivos períodos de 15 días a:
a) una HR de alrededor del $50 \%$,
b) una atmósfera saturada de humedad y
c) parcialmente sumergidas en agua destilada en botes individuales de polietileno.

\section{Procedimiento}

El método para estimar los valores de $R_{\mathrm{p}}$ en mortero endurecido a partir de la fórmula 
de Stern: $I_{\text {corr }}=B / R_{\mathrm{p}}$ ha sido ampliamente descrito en un trabajo previo (4). La constante $B$ elegida fue de $26 \mathrm{mV}$ para el acero en estado activo y $52 \mathrm{mV}$ para el estado pa. sivo.

Las medidas de $R_{\mathrm{p}}$ se realizaron todos los días con un potenciostato AMEL con "positive feed-back" que compensa instrumentalmente la caída óhmica, RI, entre el electrodo de trabajo y el de referencia, debida a la elevada resistividad del mortero.

Al final de los ensayos se determinó gravimétricamente la pérdida de peso de cada barra.

Para asegurar un buen contacto eléctrico se interpuso un papel de filtro humedecido, entre el electrodo de referencia y el mortero.

\section{Resultados}

La figura 1 reproduce el comportamiento de las tres series de probetas ensayadas, siendo cada punto la media aritmética de los valores de cuatro redondos.

Recién fabricadas las probetas tienen un comportamiento similar, con densidades de corriente de alrededor de $10 \mu \mathrm{A} / \mathrm{cm}^{2}$. Sin embargo, $24 \mathrm{~h}$ después de la fabricación ya es evidente el efecto agresivo de los $\mathrm{Cl}^{-}$. Luego, y después de un fuerte descenso, la $i_{\text {corr }}$ tiende a una progresiva estabilidad, siendo la velocidad de ataque de las probetas adicionadas de $\mathrm{Cl}^{-}$alrededor de 4-5 veces mayor que las adicionadas con $\mathrm{NO}_{2}^{-}$o sin aditivos; éstas no presentan diferencias de $i_{\text {corr }}$ significativas hasta el final del período de curado.

En la figura 2 (en la que cada punto es ahora la media de dos barras, al igual que en las figuras sucesivas), se han representado las $i_{\text {corr }}$ y $E_{\text {corr }}$ de probetas sin aditivos. Durante el período de carbonatación, la $i_{\text {corr }}$ presenta un aumento inicial rápido multiplicándose por un factor de 10, y luego cae alcanzando valores similares a los de antes de la carbonatación. El comportamiento de $E_{\text {corr }}$ es similar aunque en sentido opuesto, alcanzando al principio del período de carbonatación valores muy catódicos (activos) para luego llegar a valores más anódicos (nobles) que las probetas no carbonatadas.

En los valores posteriores al período de carbonatación de ambas variables queda patente el acusado efecto de la HR ambiental sobre las probetas carbonatadas, produciéndose aumentos considerables en la $i_{\text {corr }}$ y simultáneamente saltos hacia potenciales más negativos (catódicos) del $E_{\text {corr }}$, tanto más pronunciados cuanto mayor es el contenido en humedad del mortero. En contraste, en las probetas de control (no carbonatadas), las variaciones del contenido en agua de los poros del mortero modifican poco la $i_{\text {corr }}$ y el $E_{\text {corr }}$, que se agrupan en una banda horizontal relativamente estrecha (rayada en la figura).

En resumen, puede afirmarse, que en atmósferas secas, la carbonatación por sí sola, no supone un peligro para la estabilidad de las armaduras. Pero el riesgo es grande en atmósferas húmedas, o en estructuras parcialmente sumergidas, condiciones en las que el ataque del acero se multiplica por factores del orden de $10-10^{2}$.

Cuando se adiciona al mortero un $2 \% \mathrm{de} \mathrm{Cl}_{2} \mathrm{Ca}$, los comportamientos correspondientes a las probetas carbonatadas y sin carbonatar siguen cursos semejantes a los del mortero sin aditivos, según testimonia la figura 3. Durante la carbonatación también se produce un aumento repentino y considerable del valor de la $i_{\text {(n.r. }}$ en relación a las probetas de control, y un salto hacia valores más catódicos del $E_{\text {c.... }}$, para luego ir evolucionando ambas variables en la atmósfera del $50 \%$ de HR hasta situaciones similares a antes de la carbonatación. Los

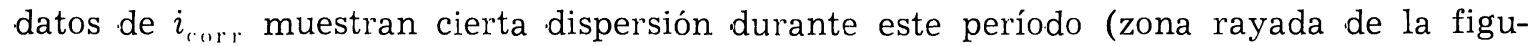


ra 3), sin embargo, es patente la tendencia con el tiempo hacia valores menores. La velocidad de corrosión es siempre más acusada en el mortero carbonatado con $\mathrm{Cl}_{2} \mathrm{Ca}$ que sin aditivos y las armaduras embebidas en mortero con $2 \%$ de $\mathrm{Cl}_{2} \mathrm{Ca}$ presentan $E_{\text {corr }}$ más negativos.

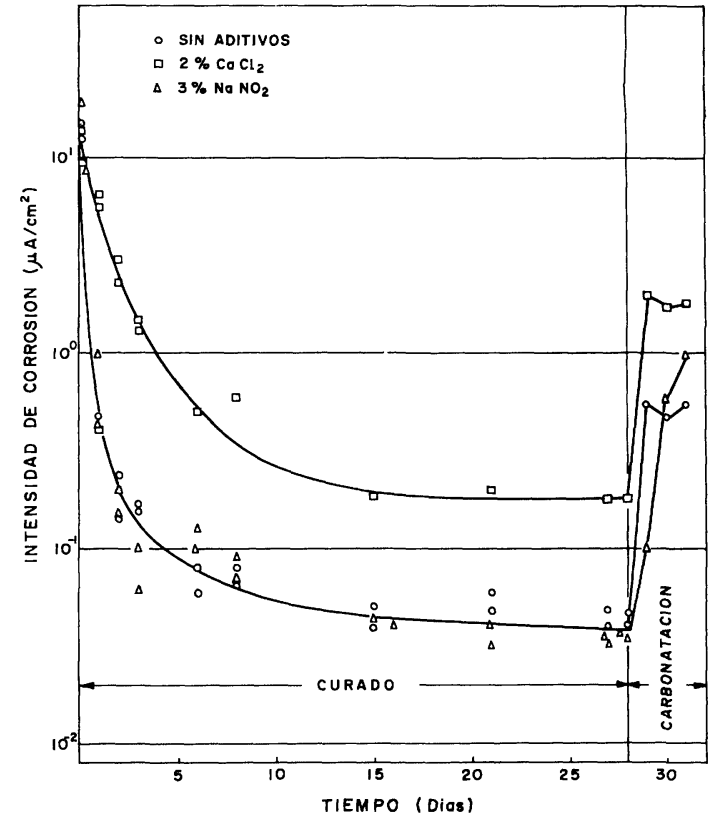

Fig. 1.-Evolución de $i_{\text {corr }}$ con el tiempo, de redondos de acero embebidos en mortero, en función del tiempo de curado y la naturaleza de los aditivos.
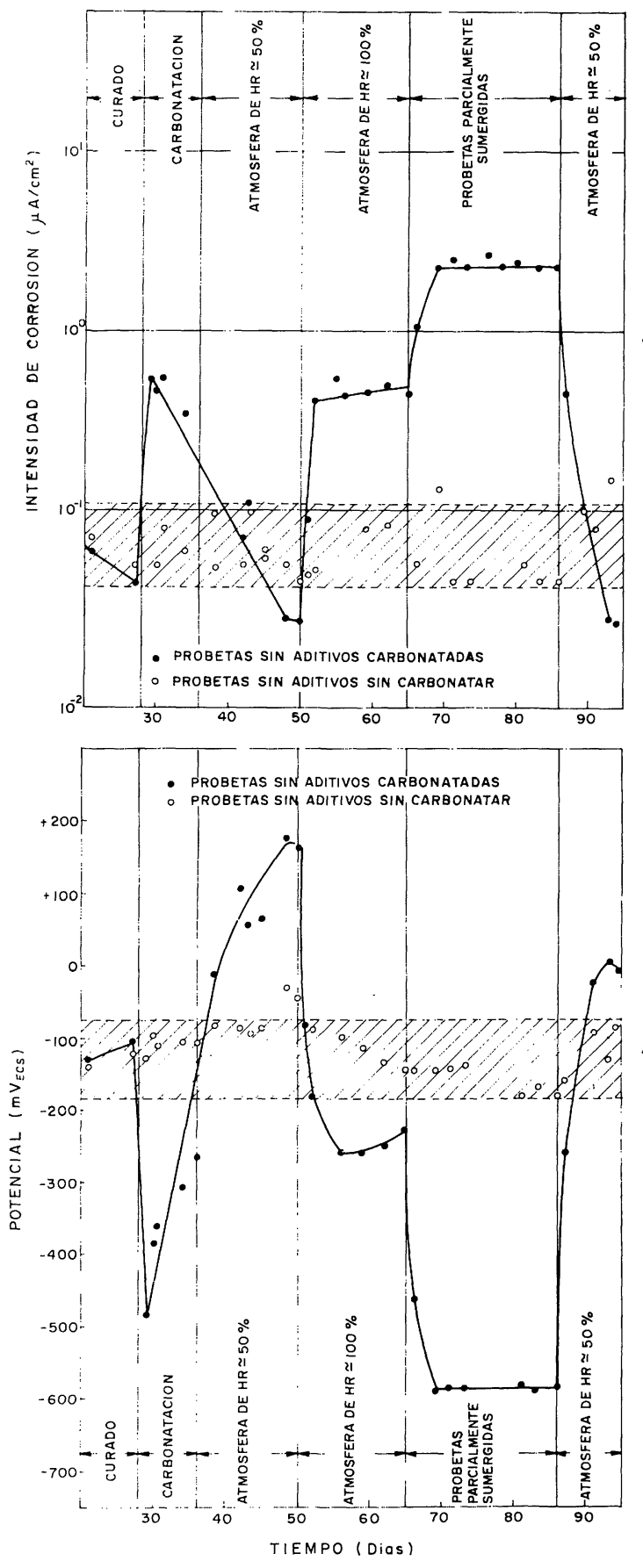

Fig. 2.-Efecto de la carbonatación y el contenido de humedad sobre $i_{\text {corr }}$ y $\boldsymbol{L}_{\text {corr }}$ de aceros embebidos en mortero sin aditivos.

En la figura 4 se han representado los comportamientos de los aceros embebidos en las probetas con $3 \%$ de $\mathrm{NO}_{2} \mathrm{Na}$. Se muestra que los valores de $i_{\text {corr }}$ y $E_{\text {corr }}$ de probetas no carbonatadas (zona rayada en figura 4) son similares a las no carbonatadas sin aditivos, mostradas en la figura 2 (zona rayada). Sin embargo, el comportamiento de las probetas 
carbonatadas es completamente diferente. Las particularidades más importantes de este comportamiento son:

a) El efecto de la carbonatación en los valores de $E_{\text {corr }}$ es opuesto al producido en mortero sin aditivos o con $\mathrm{Cl}_{2} \mathrm{Ca}$. En lugar de un salto catódico de $350-400 \mathrm{mV}$, se produce un salto en dirección anódica más pequeño.

b) Con $\mathrm{NO}_{2} \mathrm{Na}$ la dispersión de resultados es mayor.
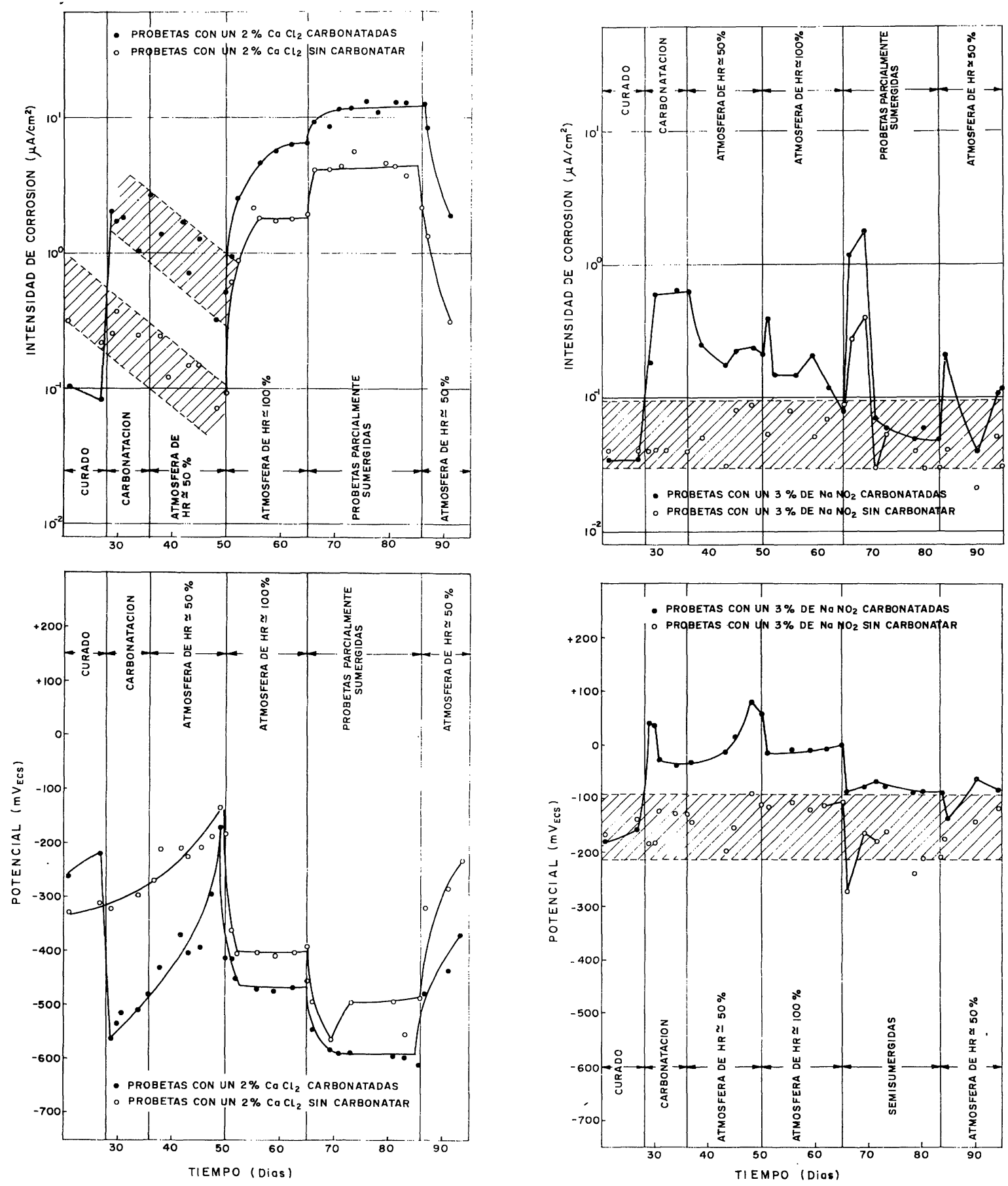

Fig. 3.-Efecto de la carbonatación y el contenido de humedad sobre $i_{\text {corr }}$ y $E_{\text {corr }}$ de aceros embebidos en mortero conteniendo $2 \%$ de $\mathrm{Cl}_{2} \mathrm{Ca}$.

Fig. 4.-Efecto de la carbonatación y el contenido de humedad sobre $i_{\text {corr }}$ y $E_{\text {corr }}$ de aceros embebidos en mortero conteniendo $3 \%$ de $\mathrm{NO}_{2} \mathrm{Na}$. 
La figura 4 muestra también que, en atmósfera seca, la adición de $\mathrm{NO}_{2} \mathrm{Na}$ tiene muy poco efecto, o incluso produce un efecto opuesto al deseado. Sin embargo, en atmósfera saturada de humedad, y fundamentalmente en inmersión parcial, el $\mathrm{NO}_{2} \mathrm{Na}$ parece inhibir la elevada velocidad de corrosión promovida por la carbonatación.

La figura 5 representa una panorámica general de la influencia de la humedad ambiental en la velocidad de corrosión de las armaduras embebidas en mortero sin y con aditivos. carbonatado y sin carbonatar. Para construir los histogramas se tomaron las $i_{\text {corr }}$ alcanzadas después de 10 días de permanencia en cada HR estudiada, es decir, cuando casi ya se ha alcanzado un estado estacionario. Se quiere resaltar cuánto puede llegar a limitar la cinética del ataque una reducida $\mathrm{HR}$ en la atmósfera, incluso cuando concurre con la carbonatación del mortero la presencia de iones cloruro en el mismo.

Los mismos aceros usados para las medidas de $R_{\mathrm{p}}$ fueron pesados antes y después (previo decapado químico) de los ensayos para comparar las pérdidas de peso obtenidas gravimétricamente con las calculadas a partir de los datos electroquímicos, y determinar la fiabilidad de estos últimos. Los resultados se dan en la figura 6. El máximo error obtenido es de alrededor de un $100 \%$, lo que según Stern y Weisert (9) representa una buena concordancia entre ambos tipos de medidas.
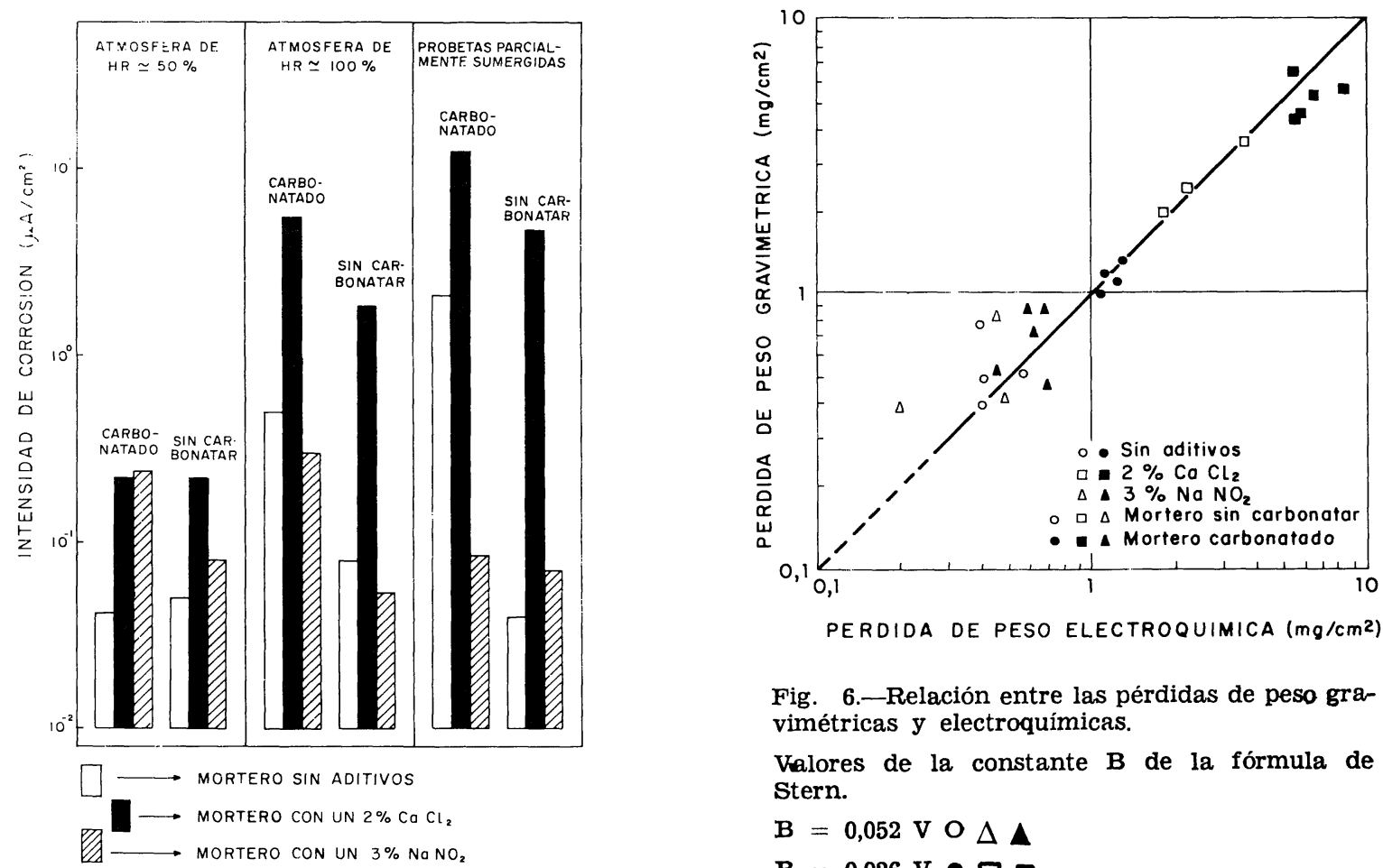

Fig. 6.-Relación entre las pérdidas de peso gravimétricas y electroquímicas.

Valores de la constante $B$ de la fórmula de Stern.

$\mathrm{B}=0,052 \mathrm{~V}$ O $\Delta \Delta$

$\mathrm{B}=0,026 \mathrm{~V} \bullet \square$

Fig. 5.-Efecto de los aditivos empleados y de las condiciones de exposición sobre la velocidad de corrosión.

La morfología del ataque se corresponde con los datos de la bibliografía, apareciendo picaduras cuando la corrosión es debida a los $\mathrm{Cl}^{-}$y corrosión generalizada, cuando está causada por la carbonatación. Cloruros y carbonatación, conjuntamente, provocan la superposición de ambos tipos de ataque, y se puede observar simultáneamente corrosión generalizada y por picaduraas con cinéticas de ataque mayores que cuando actúan ambos factores separadamente. Por otro lado, con adiciones del $3 \%$ de $\mathrm{NO}_{2} \mathrm{Na}$, la capa de pasivación sobre los redondos permanece prácticamente inalterada ; sólamente en unos pocos casos se observa un leve ataque localizado, tal vez debido a un incompleto efecto protector de los $\mathrm{NO}_{2}^{-}$. 


\section{Discusión}

\section{Mecanismo del proceso de corrosión de las armaduras en mortero carbonatado}

La fórmula de Nernst:

$$
E=E_{0}+0,059 \log a_{\mathrm{H}}+
$$

+ muestra que cuando un medio se acidifica, se produce un aumento en el $E_{\text {corr }}$ de $5^{\circ} \mathrm{mV}$ en la dirección anódica (noble) por cada unidad de variación de $\mathrm{pH}$. El cambio desde $\mathrm{pH}=13 \mathrm{a} \mathrm{pH}=7-8$ supondría un aumento de $250-300 \mathrm{mV}$.

Este es el resultado en el caso de los aceros embebidos en probetas con $\mathrm{NO}_{2} \mathrm{Na}$, cuya capa pasiva, en general, no es alterada por el proceso de carbonatación. Pero las probetas sin aditivos o con $\mathrm{Cl}_{2} \mathrm{Ca}$ presentan al contrario un salto inicial en dirección catódica. Los autores atribuyen este salto catódico a una rotura local o a la desaparición de la capa pasiva típica del medio alcalino. Luego, si el medio es suficientemente seco, las probetas sin aditivos pueden alcanzar de nuevo el estado pasivo con una baja velocidad de corrosión y valores de $E_{\text {corr }}$ similares a los obtenidos con las probetas adicionadas con $\mathrm{NO}_{2} \mathrm{Na}$. $\mathrm{Pe}-$ ro en condiciones de humedad ambiental elevada o en las probetas con adiciones de $\mathrm{Cl}^{-}$ los $E_{\text {corr }}$ se mantienen en valores catódicos (activos), entre $-200 \mathrm{y}-600 \mathrm{mV}_{\mathrm{ECS}} \mathrm{y}$ las $i_{\text {corr }}$ alcanzan valores relativamente elevados.

Considerando pues, que la carbonatación implica una rotura de la pasivación, y un desplazamiento paralelo de la rama catódica en dirección de los potenciales nobles por efecto del cambio de $\mathrm{pH}$, se ha intentado representar el fenómeno en el diagrama de Evans de la figura 7. En ella la curva 1 representa la situación previa a la carbonatación (estado pasivo), la curva 2 representa el estado carbonatado (activo), la curva 3 una pasividad menos efectiva, y los puntos $A, B, C, D$, son los representativos del sistema acero/mortero bajo las siguientes condiciones: $A$ antes de la carbonatación, $B$ después de la carbonatación en un mortero húmedo sin aditivos, $C$ después de la carbonatación en un mortero con $2 \%$ de $\mathrm{Cl}_{2} \mathrm{Ca}$ y $\mathrm{D}$ después de la carbonatación en un mortero con $3 \%$ de $\mathrm{NO}_{3} \mathrm{Na}$.

Un aumento del contenido en agua de los poros del mortero carbonatado o la adición de $\mathrm{Cl}_{2} \mathrm{Ca}$ facilita (despolariza) el proceso anódico dando lugar a una mayor velocidad de corrosión $\left(i_{\mathrm{C}}>i_{\mathrm{B}}\right)$ y a un potencial de corrosión más negativo $\left(E_{\mathrm{C}}<E_{\mathrm{B}}\right)$.

Con $3 \%$ de $\mathrm{NO}_{2} \mathrm{Na}$ se alcanza una pasividad menos perfecta que cuando no hay aditivos, aumentando ligeramente la $i_{\text {corr }}$. La situación puede ser visualizada por una rama anódica como la 3 , con un fuerte ataque, $i,>i$, a pesar del cambio simultáneo en dirección de los potenc:ales nobles del $E_{\text {corr }},\left(E_{,}>E_{A}\right)$.

Son, fundamentalmente, las modificaciones de la rama anódica las que determinan la cinética. Esto está en contradicción con la suposición generalizada de que es la difusión del $\mathrm{O}_{2}$ hasta las armaduras, lo que controla la cinética de corrosión en el hormigón carbonatado (3). Pero si esto último sucediese se alcanzaría una densidad de corriente límite independiente de variables como contenido de $\mathrm{Cl}^{-}$o humedad del mortero, lo que no parece ocurrir de acuerdo con las figuras 2-5. Tal vez, bajo otras condiciones, con un mortero menos poroso que el presente (porosidad alrededor del $14 \%$ ) y con un espesor de recubrimiento más grueso, es posible que el proceso controlante fuera la difusión del $\mathrm{O}_{2}$.

Otra conclusión se puede deducir a partir de estos resultados: en un mortero carbonatado con valores de $\mathrm{pH}$ próximos a la neutralıdad, la amplitud de la variación de $E_{\text {curr }}$ observada ( +200 a $-600 \mathrm{mV}$, fig. 2 y 3 ) indica que no sólo es posible la corrosión generalizada sino que también la pasividad puede tener lugar. 


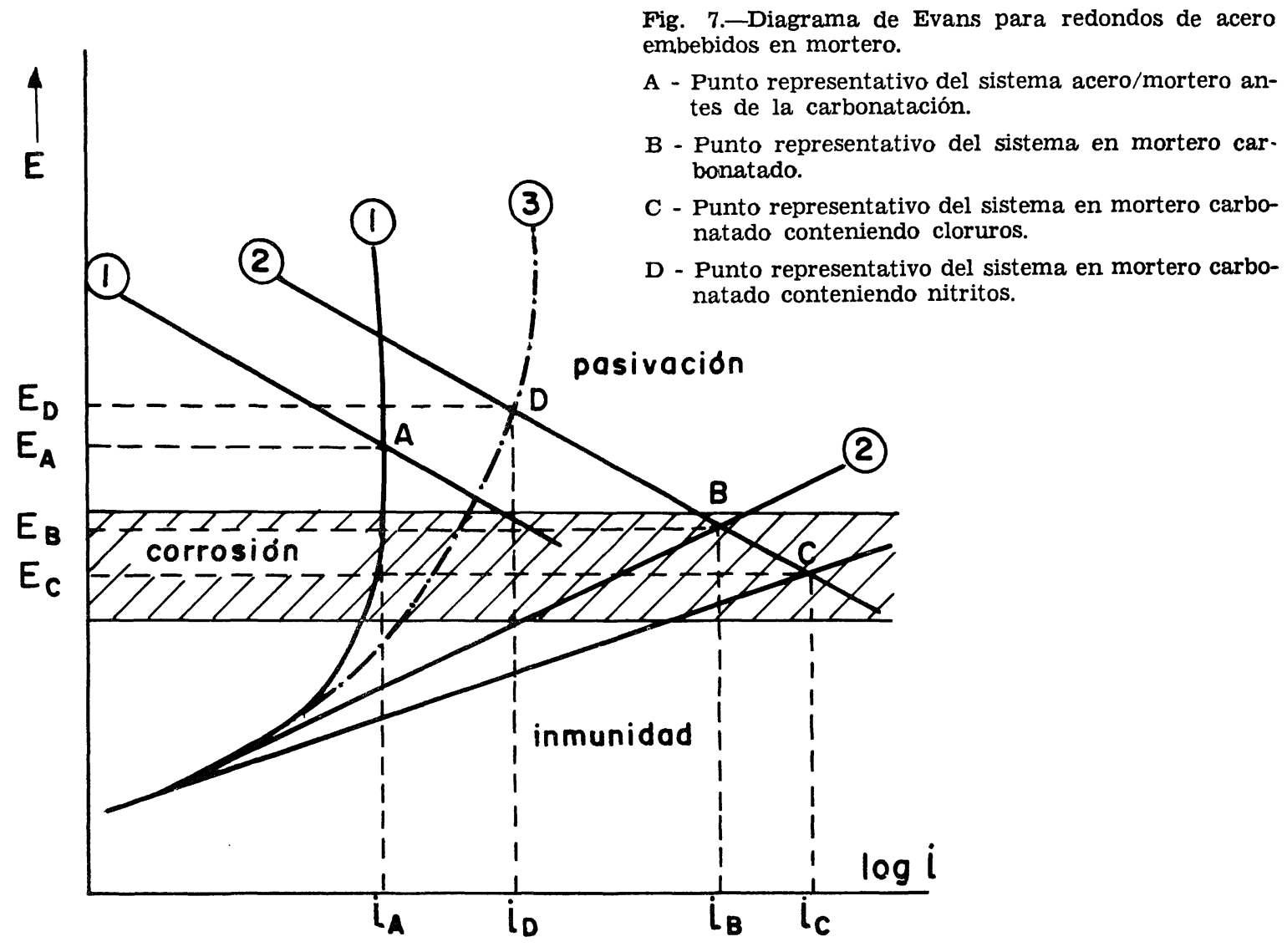

\section{Efecto combinado de la carbonatación, aditivos y humedad relativa sobre la velocidad de corrosión de las armaduras}

Las figuras 2-5 confirman el conocimiento general de que sólo es preocupante el ataque a las armaduras cuando el mortero está carbonatado y/o contiene iones despasivantes. Bajo tales condiciones, el contenido en agua de los poros del hormigón es el factor determinante de la cinética de ataque.

Así, la información dada por las anteriores figuras supone un avance considerable al mostrar la cuantía de los efectos de cada factor de corrosión que, hasta ahora, sólo se podían apreciar cualitativamente. Por ejemplo, en amósferas saturadas de humedad, el acero sufre un ataque 10 veces mayor en mortero carbonatado con $2 \% \mathrm{Cl}_{2} \mathrm{Ca}$, que sin este aditivo o 3-4 veces mayor que en mortero no-carbonatado con $2 \% \mathrm{Cl}_{2} \mathrm{Ca}$ (fig. 5). En condiciones de inmersión parcial esta agresividad relativa se mantiene, aunque las relaciones cuantitativas son algo diferentes.

Asimismo, se demuestra que el mortero que no contiene cloruros y no está carbonatado, es suficientemente protector para las armaduras cualquiera que sea el contenido en humedad del hormigón. En tales condiciones la adición de $\mathrm{NO}_{2} \mathrm{Na}$ no es necesaria. Pero, la mejora conseguida con los nitritos en condiciones muy agresivas como la inmersión parcial (figs. 4 y 5), es suficientemente prometedora para animar a futuros estudios sobre la proporción óptima de $\mathrm{NO}_{2} \mathrm{Na}$ y la duración de su efecto en hormigones carbonatados.

\section{Fiabilidad de las estimaciones electroquímicas}

Las evaluaciones electroquímicas serían más exactas si cada probeta se hubiera conserva- 
do todo el tiempo en un ambiente con la misma humedad relativa, ya que la constante $B$ de la fórmula de Stern puede ser que varíe, con el contenido en humedad de la atmósfera.

A pesar de esta lim:tación, la técnica de medida de la $R_{\mathrm{p}}$ ha proporcionado resultados fiables, pues las desviaciones observadas resultan insignificantes cuando se comparan con el rango de variación de $i_{c, r r}\left(\mathrm{de} \sim 10^{-2}\right.$ a $\left.10 \mu \mathrm{A} / \mathrm{cm}^{2}\right)$ encontrado en las condiciones del ensayo.

La $R_{\mathrm{p}}$ como técnica de medida se ha mostrado como una herramienta muy útil para una evaluación rápida, cuantitativa y no destructiva, del efecto de la carbonatación del mortero, de sus aditivos y de la humedad, sobre la $i_{\text {corr }}$ de las armaduras.

\section{O N C L U S I O N E S}

De los resultados observados se pueden extraer las siguientes conclusiones:

1.") La carbonatación del cemento no provoca, por sí misma, una corrosión apreciable de las armaduras.

Para producir una velocidad de corrosión notable, es necesario que la HR ambiental sea superior a un mínimo crítico $(>50 \%)$, que es de esperar que dependa del tipo de mortero y de los aditivos empleados.

2.') La adición de un $2 \%$ de $\mathrm{Cl}_{2} \mathrm{Ca}$ al mortero carbonatado aumenta la velocidad de corrosión.

Por el contrario, el ataque es considerablemente reducido por la adición de un $3 \%$ de $\mathrm{NO}_{2} \mathrm{Na}$, incluso en atmósferas saturadas de humedad o en condiciones de inmersión parcial.

3.") El método de la $R_{\mathrm{p}}$ ha demostrado ser una técnica cuantitativa fiable y rápida para la medida de $i_{\text {corr }}$ en armaduras embebidas en hormigón, y ha revelado una buena concordancia entre los datos electroquímicos obtenidos y el ataque real que diferentes factores de corrosión provocan sobre las armaduras.

\section{B I B L I O G R A F I A}

(1) Venuat, M.: Mater. et Const. 1978, 11, 142.

(2) Stolte, E., y Bohnenkamp, K.: Korrosionschutz metallischer Werkstoffe im Hoch und Ingenierbau, 1976, pg. 113-127 (Düsseldorf : Verlag-Stahleisen M.B.H.).

(3) RILEM (12-CRC Committee): Mater. et Const, 1976, 9, 187.

(4) Andrade, C. y Gonzalez, J. A.: Werkstoffe Korros, 1978, 29, 515.

(5) KAESCHE, H.: “Zement-Kalk-Gips”, 1959, 12, 289.

(G) BAUMEL, A.: Ibid. 1959, 12, 294.

(7) Baumel, A., y Engell, H. J.: Arch. Eisenhüitten Wes., 1959, 30, 417.

(8) Stern, M. y Geary, A. L.: J. Electrochem. Soc., 1957, 104, 56.

(9) Stern, M. y Weisert, E. D. : Proc. Am. Soc. Test. Mater., 1959, 59, 1.280

(10) RC-75 Pliego de prescripciones técnicas generales para la recepción de cemento, 1975 - MOPU, Madrid. 